\title{
Bisimulations in fuzzy social network analysis
}

\author{
Jelena Ignjatović, Miroslav Ćirić, Ivan Stanković \\ University of Niš, Faculty of Sciences and Mathematics, Višegradska 33, 18000 Niš, Serbia
}

\begin{abstract}
In this paper we introduce two types of simulations and five types of bisimulations for fuzzy social networks, and we study one of them - regular bisimulations. We prove that if there exists at least one regular bisimulation between two fuzzy networks, then there exists the greatest bisimulation of this type, and we provide a procedure for testing the existence of a regular bisimulation between two fuzzy networks, and computing the greatest one, whenever it exists. We also establish a natural relationship between regular bisimulations and regular fuzzy equivalences.
\end{abstract}

Keywords: Fuzzy social network, regular bisimulation, regular fuzzy equivalence, uniform fuzzy relation, fuzzy relation equation, residual of fuzzy relations.

\section{Introduction}

Positional analysis is a branch of the social network analysis whose main aim is to find structural similarities between actors which have to reflect their position or role in a network. These similarities were first formalized by Lorrain and White [20] by the concept of a structural equivalence, where two actors are considered to be structurally equivalent if they have identical neighborhoods. In many cases this concept has shown oneself to be too strong, and White and Reitz [26] introduced the concept of a regular equivalence as more appropriate for modeling social positions. In this case, two actors are considered to be regularly equivalent if they are equally related to equivalent others. Afterwards, regular equivalences were studied in numerous papers, e.g., in $[1,11,12,24]$.

Social relations are essentially vague so it is quite natural that few authors initiated the study of social networks from the aspect of the theory of fuzzy sets (cf. [5, 13, 14, 15, 17, 18, 19]). In the study of fuzzy social networks conducted in the listed papers positional analysis and regular fuzzy equivalences had a central place. Regular fuzzy equivalences have been first studied by Fan et al. [14, 15], where they were called regular similarities, and from a similar point of view they have been recently investigated in [13]. From a different point of view regular fuzzy equivalences have been studied in $[5,17,18,19]$, and there it has been shown that the greatest regular fuzzy equivalence on a fuzzy network can be computed as the greatest solution to a particular system of fuzzy relation equations. Such an approach has previously been shown to be very efficient in solving some fundamental problems of the theory of fuzzy automata, such as the reduction of the number of states and the problems of equivalence, simulation and bisimulation (cf. [7, 8, 9, 10, 17, 25]).

As we have already said, the role of regular equivalences is to establish some kind of structural similarities between actors in a network as the basis for partitioning a network into sets of actors which occupy the same social position. In the present paper we discuss a somewhat different problem: How to find the same kind of structural similarities between actors from two different networks, as the basis for identifying related positions in these networks? This problem has already been discussed by Marx and Masuch [21], who pointed to the concept of a bisimulation that has been successfully used for similar purposes in some related areas of computer science and mathematics.

Bisimulations have been introduced by Milner in [22] and Park in [23] in computer science, more precisely in concurrency theory. Roughly at the same time they have been also discovered in some areas of mathematics, e.g., in modal logic and set theory. They are widely used to model equivalence between various systems, as well as to reduce the number of states of these systems, and are employed today in many areas of computer science. A few papers dealt with bisimulations in the context of social networks. Marx and Masuch [21] have defined what we call here a forward bisimulation between two networks, and have equated regular equivalences with what they have called in-out bisimulations. However, they have dealt mainly with bisimulations that relate actors from the same network and have not conducted a deeper study of bisimulations between different networks. Forward bisimulations between two networks have been also mentioned in [13], but again, they have not been deeply investigated. And finally, Brynielsson et al. [4] have studied what we call forward and backward simulations, but also as relations between the actors from the same network, and they have not discussed simulations between different networks.

In this paper we perform a deeper study of bisimulations between two different fuzzy social networks. We define two types of simulations and five types of bisimulations, including the regular bisimulations, which are the main subject of the paper. We prove that if there exists at least one regular bisimulation between two fuzzy networks, then there exists the 
greatest bisimulation of this type (Theorem 3.1). Then we provide a procedure by which both we test the existence of a regular bisimulation between two fuzzy networks, and compute the greatest one, whenever it exists (Theorems 3.2 and 3.3).

In addition to bisimulations, another very important concept that we use in our research is that of a uniform fuzzy relation. Uniform fuzzy relation have been introduced in [6], and the original intention of the authors was to introduce them as a basis for defining such concept of a fuzzy function which would provide a correspondence between fuzzy functions and fuzzy equivalence relations, analogous to the correspondence between crisp functions and crisp equivalence relations. This was done, but also, it turned out that uniform fuzzy relations establish natural relationships between fuzzy partitions of two sets, some kind of "uniformity" between these fuzzy partitions. Roughly speaking, uniform fuzzy relations can be conceived as fuzzy equivalence relations which relate elements of two possibly different sets, and from this point of view, they can be very successfully used in conjunction with bisimulations, which have a complementary role. For this reason, we consider uniform regular bisimulations between two networks, and by Theorem 3.4 we show that any uniform regular bisimulation determines a pair of regular equivalences on these networks and a particular isomorphism between the corresponding quotient fuzzy networks (i.e., an isomorphism between the positions in these networks), and vice versa.

\section{Preliminaries}

\subsection{Fuzzy sets and fuzzy relations}

In the paper we use complete residuated lattices as structures of membership values. A residuated lattice is an algebra $\mathcal{L}=(L, \wedge, \vee, \otimes, \rightarrow, 0,1)$ such that

(L1) $(L, \wedge, \vee, 0,1)$ is a lattice with the least element 0 and the greatest element 1 ,

(L2) $(L, \otimes, 1)$ is a commutative monoid with the unit 1

(L3) $\otimes$ and $\rightarrow$ form an adjoint pair, i.e., they satisfy the adjunction property: for all $x, y, z \in L$,

$$
x \otimes y \leqslant z \Leftrightarrow x \leqslant y \rightarrow z .
$$

If, additionally, $(L, \wedge, \vee, 0,1)$ is a complete lattice, then $\mathcal{L}$ is called a complete residuated lattice.

The operations $\otimes$ (called multiplication) and $\rightarrow$ (called residuum) are intended for modeling the conjunction and implication of the corresponding logical calculus, and supremum $(\bigvee)$ and infimum $(\bigwedge)$ are intended for modeling the existential and general quantifier, respectively. An operation $\leftrightarrow$ given by

$$
x \leftrightarrow y=(x \rightarrow y) \wedge(y \rightarrow x),
$$

called biresiduum (or biimplication), is used for modeling the equivalence of truth values. It can be easily shown that with respect to $\leqslant, \otimes$ is isotonic in both arguments, $\rightarrow$ is isotonic in the second and antitonic in the first argument. For other properties of complete residuated lattices we refer to $[2,3]$.

The most studied and applied structures of membership values, defined on the real unit interval $[0,1]$ with $x \wedge y=\min (x, y)$ and $x \vee y=\max (x, y)$, are the Lukasiewicz structure $(x \otimes y=\max (x+y-1,0)$ and $x \rightarrow y=\min (1-x+y, 1))$, the Gödel structure $(x \otimes y$ $=\min (x, y), x \rightarrow y=1$ if $x \leqslant y$ and $x \rightarrow y=y$ otherwise), and the Goguen or product structure $(x \otimes y=x \cdot y, x \rightarrow y=1$ if $x \leqslant y$ and $x \rightarrow y$ $=y / x$ otherwise). Another important set of truth values is the set $\left\{a_{0}, a_{1}, \ldots, a_{n}\right\}, 0=a_{0}<\cdots<$ $a_{n}=1$, with $a_{k} \otimes a_{l}=a_{\max (k+l-n, 0)}, a_{k} \rightarrow a_{l}=$ $a_{\min (n-k+l, n)}$. A special case of the latter algebras is the two-element Boolean algebra of classical logic with the support $\{0,1\}$. The only adjoint pair on this Boolean algebra consists of the classical conjunction and implication operations. This structure of truth values is called the Boolean structure.

In the sequel $\mathcal{L}$ will be a complete residuated lattice. A fuzzy subset of a set $A$ over $\mathcal{L}$, or simply a fuzzy subset of $A$, is any mapping from $A$ into $L$. Ordinary crisp subsets of $A$ are considered as fuzzy subsets of $A$ taking membership values in the set $\{0,1\} \subseteq L$. Let $f$ and $g$ be two fuzzy subsets of $A$. The equality of $f$ and $g$ is defined as the usual equality of mappings, i.e., $f=g$ if and only if $f(x)=g(x)$, for every $x \in A$. The inclusion $f \leqslant g$ is also defined pointwise: $f \leqslant g$ if and only if $f(x) \leqslant g(x)$, for every $x \in A$. Endowed with this partial order the set $L^{A}$ of all fuzzy subsets of $A$ forms a complete lattice, in which the meet (intersection) $\bigwedge_{i \in I} f_{i}$ and the join (union) $\bigvee_{i \in I} f_{i}$ of an arbitrary family $\left\{f_{i}\right\}_{i \in I}$ of fuzzy subsets of $A$ are mappings from $A$ into $L$ defined by

$$
\left(\bigwedge_{i \in I} f_{i}\right)(x)=\bigwedge_{i \in I} f_{i}(x), \quad\left(\bigvee_{i \in I} f_{i}\right)(x)=\bigvee_{i \in I} f_{i}(x)
$$

for all $x \in A$.

A fuzzy relation between sets $A$ and $B$ (in this order) is any fuzzy subset of $A \times B$, and the equality, inclusion (ordering), joins and meets of fuzzy relations are defined as for fuzzy sets. The set of all fuzzy relations between $A$ and $B$ will be denoted by $L^{A \times B}$. In particular, a fuzzy relation on a set $A$ is any fuzzy subset of $A \times A$, and the set of all fuzzy relations on $A$ is denoted by $L^{A \times A}$. The reverse or inverse of a fuzzy relation $\varphi \in L^{A \times B}$ is a fuzzy relation $\varphi^{-1} \in L^{B \times A}$ defined by $\varphi^{-1}(b, a)=\varphi(a, b)$, for all $a \in A$ and $b \in B$.

For non-empty sets $A, B$ and $C$, and fuzzy relations $\varphi \in L^{A \times B}$ and $\psi \in L^{B \times C}$, their composition is a fuzzy relation $\varphi \circ \psi \in L^{A \times C}$ defined by

$$
(\varphi \circ \psi)(a, c)=\bigvee_{b \in B} \varphi(a, b) \otimes \psi(b, c),
$$

for all $a \in A$ and $c \in C$. Moreover, for a fuzzy relation $\varphi \in L^{A \times B}$ and fuzzy sets $f \in L^{A}$ and $g \in L^{B}$, 
we define a fuzzy set $f \circ \varphi \in L^{B}$ by

$$
(f \circ \varphi)(b)=\bigvee_{a \in A} f(a) \otimes \varphi(a, b),
$$

for each $b \in B$, and a fuzzy set $\varphi \circ g \in L^{A}$ by

$$
(\varphi \circ g)(a)=\bigvee_{b \in b} \varphi(a, b) \otimes g(b),
$$

for each $a \in A$. In both cases, the operation $\circ$ is called the composition of a fuzzy relation and a fuzzy set. It is easy to verify that $f \circ \varphi=\varphi^{-1} \circ f$ and $\varphi \circ g=g \circ \varphi^{-1}$. For fuzzy sets $f, g \in L^{A}$ a scalar $f \circ g \in L$ is defined by

$$
f \circ g=\bigvee_{a \in A} f(a) \otimes g(a) .
$$

When the underlying sets are finite, fuzzy relations and fuzzy sets can be respectively interpreted as matrices and vectors with entries in $L$, the composition of fuzzy relations can be interpreted as the matrix product, compositions of a fuzzy relation and a fuzzy set as matrix-vector products, and the composition of two fuzzy sets as the scalar (dot) product of vectors. It is easy to check that the composition of fuzzy relations is associative and distributive over unions (joins) of fuzzy relations. Furthermore, for all $f \in L^{A}, g \in L^{B}$ and $\varphi \in L^{A \times B}$ we have

$$
(f \circ \varphi) \circ g=f \circ(\varphi \circ g),
$$

and both sides in the above equality will be denoted simply by $f \circ \varphi \circ g$ without using the parentheses.

Let $A, B$ and $C$ be non-empty sets, $\lambda \in L^{A \times B}$, $\mu \in L^{B \times C}$ and $\eta \in L^{A \times C}$. The right residual of $\eta$ by $\lambda$ is a fuzzy relation $\lambda \backslash \eta \in L^{B \times C}$ defined by

$$
(\lambda \backslash \eta)(b, c)=\bigwedge_{a \in A} \lambda(a, b) \rightarrow \eta(a, c)
$$

for all $(b, c) \in B \times C$, and the left residual of $\eta$ by $\mu$ is a fuzzy relation $\eta / \mu \in L^{A \times B}$ defined by

$$
(\eta / \mu)(a, b)=\bigwedge_{c \in C} \mu(b, c) \rightarrow \eta(a, c),
$$

for all $(a, b) \in A \times B$. It is not hard to verify that the following residuation property (in some sources called the adjunction property) holds for arbitrary $\lambda \in L^{A \times B}, \mu \in L^{B \times C}$ and $\eta \in L^{A \times C}$ :

$$
\lambda \circ \mu \leqslant \eta \quad \Leftrightarrow \quad \mu \leqslant \lambda \backslash \eta \quad \Leftrightarrow \quad \lambda \leqslant \eta / \mu .
$$

For fuzzy sets $f \in L^{A}$ and $g \in L^{B}$ the right residual of $g$ by $f$ is a fuzzy relation $f \backslash g \in L^{A \times B}$ defined by

$$
(f \backslash g)(a, b)=f(a) \rightarrow g(b),
$$

for all $(a, b) \in A \times B$, and the left residual of $g$ by $f$ is a fuzzy relation $g / f \in L^{B \times A}$ defined by

$$
(g / f)(b, a)=f(a) \rightarrow g(b),
$$

for all $(b, a) \in B \times A$. It is clear that $g / f=(f \backslash g)^{-1}$. For all $\varphi \in L^{A \times B}, \psi \in L^{B \times A}, f \in L^{A}$ and $g \in L^{B}$ the following residuation properties hold:

$$
f \circ \varphi \leqslant g \Leftrightarrow \varphi \leqslant f \backslash g, \quad \psi \circ f \leqslant g \Leftrightarrow \psi \leqslant g / f .
$$

A fuzzy relation $\alpha \in L^{A \times A}$ is reflexive if $\Delta_{A} \leqslant \alpha$ (where $\Delta_{A}$ is the crisp equality on $A$ ), symmetric if $\alpha^{-1} \leqslant \alpha$, and transitive if $\alpha \circ \alpha \leqslant \alpha$. A reflexive, symmetric and transitive fuzzy relation is called a fuzzy equivalence, and a reflexive and transitive fuzzy relation is called a fuzzy quasi-order. Note that if $\alpha$ is a fuzzy quasi-order, then $\alpha \circ \alpha=\alpha$. For a fuzzy equivalence $\alpha \in L^{A \times A}$ and an element $a \in A$, the fuzzy set $\alpha_{a} \in L^{A}$ defined by $\alpha_{a}(b)=\alpha(a, b)$, for each $b \in A$, is called the equivalence class of $\alpha$ determined by $a$, and $a$ is called the representative of the equivalence class $\alpha_{a}$. The set of all equivalence classes of $\alpha$ is denoted by $A / \alpha$ and called the quotient set (or factor set) of $A$ with respect to $\alpha$.

Hereinafter, $\mathbb{N}$ denotes the set of natural numbers (without zero). A partially ordered set $P$ is said to satisfy the descending chain condition, shortly $D C C$, if each descending sequence of elements of $P$ stabilizes, i.e., if $\left\{a_{k}\right\}_{k \in \mathbb{N}}$ is a sequence of elements of $P$ such that $a_{k+1} \leqslant a_{k}$, for each $k \in \mathbb{N}$, then there exists $n \in \mathbb{N}$ such that $a_{n}=a_{n+m}$, for each $m \in \mathbb{N}$.

For more information on fuzzy sets and fuzzy relations we refer to $[2,3]$.

\subsection{Uniform fuzzy relations}

Given non-empty sets $A$ and $B$ and a fuzzy relation $\varphi \in L^{A \times B}$. A fuzzy equivalence $E_{A}^{\varphi}$ on $A$ defined by

$$
E_{A}^{\varphi}\left(a, a^{\prime}\right)=\bigwedge_{b \in B} \varphi(a, b) \leftrightarrow \varphi\left(a^{\prime}, b\right)
$$

for all $a, a^{\prime} \in A$, is called the kernel of $\varphi$, and a fuzzy equivalence $E_{B}^{\varphi}$ on $B$ defined by

$$
E_{B}^{\varphi}\left(b, b^{\prime}\right)=\bigwedge_{a \in A} \varphi(a, b) \leftrightarrow \varphi\left(a, b^{\prime}\right),
$$

for all $b, b^{\prime} \in B$, is called the co-kernel of $\varphi$. If for each $a \in A$ there is $b \in B$ such that $\varphi(a, b)=1$, then $\varphi$ is called an $\mathcal{L}$-function. Note that $\varphi$ is an $\mathcal{L}$-function if and only if there is a function $\psi: A \rightarrow B$ such that $\varphi(a, \psi(a))=1$, for every $a \in A$. A function $\psi$ with this property is called a crisp description of $\varphi$. The set of all such functions is denoted by $C R(\varphi)$. If for every $b \in B$ there is $a \in A$ such that $\varphi(a, b)=1$, then $\varphi$ is called a surjective fuzzy relation.

If $\varphi \circ \varphi^{-1} \circ \varphi \leqslant \varphi$, then we call $\varphi$ a partial fuzzy function, and a partial fuzzy function which is a surjective $\mathcal{L}$-function we call a uniform fuzzy relation.

Let $A$ and $B$ be non-empty sets and $\varphi \in L^{A \times B}$ a uniform fuzzy relation, and let $\alpha=E_{A}^{\varphi}$ and $\beta=E_{B}^{\varphi}$. Then a function $\bar{\varphi}: A / \alpha \rightarrow B / \beta$ defined by

$$
\bar{\varphi}\left(\alpha_{a}\right)=\beta_{\psi(a)}, \quad \text { for any } a \in A \text { and } \psi \in C R(\varphi) \text {, }
$$


is well-defined (it does not depend on the choice of $a \in A$ and $\psi \in C R(\varphi))$ and it is a bijective function with

$$
(\bar{\varphi})^{-1}=\overline{\varphi^{-1}} .
$$

Note that the function $\bar{\varphi}$ establishes some kind of "uniformity" between the equivalence classes of the kernel and the co-kernel of $\varphi$, and from this reason uniform fuzzy relations obtained in [6] this name.

\subsection{Fuzzy networks}

A fuzzy social network is considered here as a triple $\mathcal{N}=\left(A,\left\{R_{i}\right\}_{i \in I},\left\{p_{j}\right\}_{j \in J}\right)$, where $A$ is a non-empty set, whose members are called actors, $\left\{R_{i}\right\}_{i \in I}$ is a collection of fuzzy relations on $A$, and $\left\{p_{j}\right\}_{j \in J}$ is a collection of fuzzy subsets of $A$. We assume that all sets $A, I$ and $J$, fuzzy relations $R_{i}$ and fuzzy sets $p_{j}$ are non-empty. The membership degree of an actor to a fuzzy set $p_{j}$ is understood as the degree to which this actor has a certain attribute associated to $p_{j}$. For the sake of simplicity, we will say just 'fuzzy network' instead of 'fuzzy social network'.

Two fuzzy networks $\mathcal{N}=\left(A,\left\{R_{i}\right\}_{i \in I},\left\{p_{j}\right\}_{j \in J}\right)$ and $\mathcal{N}^{\prime}=\left(A^{\prime},\left\{R_{i}^{\prime}\right\}_{i \in I},\left\{p_{j}^{\prime}\right\}_{j \in J}\right)$ are called isomorphic if there exists a bijective function $\phi: A \rightarrow A^{\prime}$ such that $R_{i}(a, b)=R_{i}^{\prime}(\phi(a), \phi(b))$, for all $a, b \in A$ and $i \in I$, and $p_{j}(a)=p_{j}^{\prime}(\phi(a))$, for all $a \in A$ and $j \in J$.

Let $\mathcal{N}=\left(A,\left\{R_{i}\right\}_{i \in I},\left\{p_{j}\right\}_{j \in J}\right)$ be a fuzzy network and $\alpha \in L^{A \times A}$ a fuzzy equivalence, and let $A / \alpha$ be the corresponding factor set. For each $i \in I$ and each $j \in J$ we define a fuzzy relation $\bar{R}_{i} \in L^{(A / \alpha) \times(A / \alpha)}$ and a fuzzy set $\bar{p}_{j} \in L^{A / \alpha}$ by

(i) $\bar{R}_{i}\left(\alpha_{a}, \alpha_{b}\right)=\left(\alpha \circ R_{i} \circ \alpha\right)(a, b)=\alpha_{a} \circ R_{i} \circ \alpha_{b}$, for all $a, b \in A$,

(ii) $\bar{p}_{j}\left(\alpha_{a}\right)=\left(\alpha \circ p_{j}\right)(a)=\alpha_{a} \circ p_{j}$, for all $a \in A$.

It is clear that all $\bar{R}_{i}$ and $\bar{p}_{j}$ are well-defined, in the sense that they do not depend on the choice of representatives of the equivalence classes of $\alpha$, and $\mathcal{N} / \alpha=\left(A / \alpha,\left\{\bar{R}_{i}\right\}_{i \in I},\left\{\bar{p}_{j}\right\}_{j \in J}\right)$ is also a fuzzy network called the quotient (or factor) fuzzy network of $\mathcal{N}$ with respect to $\alpha$. It is worth noting that a quotient fuzzy network with respect to $\alpha$ is also known as the blockmodel or blockomodel image of $\mathcal{N}$ corresponding to $\alpha$ (cf. $[1,11,12,21])$.

\section{Regular bisimulations}

Given fuzzy networks $\mathcal{N}=\left(A,\left\{R_{i}\right\}_{i \in I},\left\{p_{j}\right\}_{j \in J}\right)$ and $\mathcal{N}^{\prime}=\left(A^{\prime},\left\{R_{i}^{\prime}\right\}_{i \in I},\left\{p_{j}^{\prime}\right\}_{j \in J}\right)$, and a non-empty fuzzy relation $\varphi \in L^{A \times A^{\prime}}$. If $\varphi$ satisfies

$$
\begin{array}{cc}
p_{j} \leqslant \varphi \circ p_{j}^{\prime}, & \text { for each } j \in J, \\
p_{j} \circ \varphi \leqslant p_{j}^{\prime}, & \text { for each } j \in J, \\
\varphi^{-1} \circ R_{i} \leqslant R_{i}^{\prime} \circ \varphi^{-1}, & \text { for each } i \in I,
\end{array}
$$

then it is called a forward simulation between $\mathcal{N}$ and $\mathcal{N}^{\prime}$, and if it satisfies (10), (11) and

$$
R_{i} \circ \varphi \leqslant \varphi \circ R_{i}^{\prime}, \quad \text { for each } i \in I,
$$
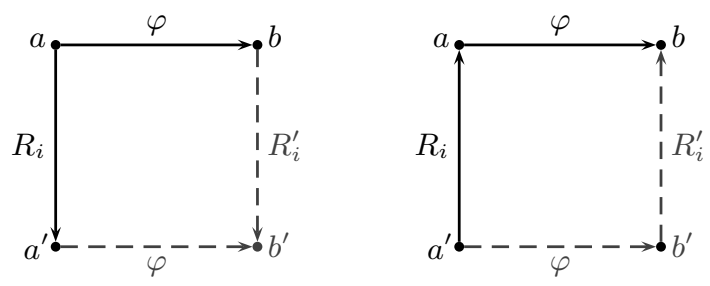

Figure 1: Forward simulation (the condition (12), on the left) and backward simulation (the condition (13), on the right).

then it is called a backward simulation between $\mathcal{N}$ and $\mathcal{N}^{\prime}$. Using these two types of simulation, we define four types of bisimulation. If both $\varphi$ and $\varphi^{-1}$ are forward simulations then $\varphi$ is called a forward bisimulation between $\mathcal{N}$ and $\mathcal{N}^{\prime}$, if $\varphi$ and $\varphi^{-1}$ are backward simulations then $\varphi$ is a backward bisimulation, if $\varphi$ is a forward and $\varphi^{-1}$ a backward simulation then $\varphi$ is a forward-backward bisimulation, and if $\varphi$ is a backward and $\varphi^{-1}$ a forward simulation then $\varphi$ is a backward-forward bisimulation. Finally, if $\varphi$ and $\varphi^{-1}$ are both forward and backward bisimulations, then $\varphi$ is called a regular bisimulation. In other words, $\varphi$ is a regular bisimulation if it satisfies

$$
\begin{array}{cc}
p_{j}=\varphi \circ p_{j}^{\prime}, & \text { for each } j \in J, \\
p_{j}^{\prime}=\varphi^{-1} \circ p_{j}, & \text { for each } j \in J, \\
R_{i} \circ \varphi=\varphi \circ R_{i}^{\prime}, & \text { for each } i \in I, \\
\varphi^{-1} \circ R_{i}=R_{i}^{\prime} \circ \varphi^{-1}, & \text { for each } i \in I .
\end{array}
$$

The meaning of simulations and bisimulations can be well explained in the case when $\mathcal{N}$ and $\mathcal{N}^{\prime}$ are crisp (Boolean-valued) networks. The condition (11) means that if an actor $a \in A$ has a certain attribute and it is simulated by some actor $b \in B$, then $b$ has the same attribute, whereas the condition (10) means that any actor $a \in A$ having a certain attribute can be simulated by an actor $b \in B$ with the same attribute. The meaning of the conditions (12) and (13) is shown in Fig. 1.

In this paper our attention will be entirely focused on the most restrictive type of bisimulations, on regular bisimulations.

First we prove the following theorem.

Theorem 3.1 Let $\mathcal{N}$ and $\mathcal{N}^{\prime}$ be fuzzy networks such that there exists at least one regular bisimulation between $\mathcal{N}$ and $\mathcal{N}^{\prime}$. Then there exists the greatest regular bisimulation between $\mathcal{N}$ and $\mathcal{N}^{\prime}$.

In addition, the greatest regular bisimulation between $\mathcal{N}$ and $\mathcal{N}^{\prime}$ is a partial fuzzy function.

Proof. If the family of all regular bisimulations between $\mathcal{N}$ and $\mathcal{N}^{\prime}$ is non-empty, it is easy to check that the union of this family is also a regular bisimulation, and it is the greatest regular bisimulation between $\mathcal{N}$ and $\mathcal{N}^{\prime}$.

Next, if $\varphi$ is the greatest regular bisimulation between $\mathcal{N}$ and $\mathcal{N}^{\prime}$, then $\varphi \circ \varphi^{-1} \circ \varphi$ is also a regular bisimulaton between $\mathcal{N}$ and $\mathcal{N}^{\prime}$, and we conclude that $\varphi \circ \varphi^{-1} \circ \varphi \leqslant \varphi$. Therefore, $\varphi$ is a partial fuzzy function. 
The second theorem provides a method for testing the existence of a regular bisimulation between two fuzzy networks.

Theorem 3.2 Let $\mathcal{N}=\left(A,\left\{R_{i}\right\}_{i \in I},\left\{p_{j}\right\}_{j \in J}\right)$ and $\mathcal{N}^{\prime}=\left(A^{\prime},\left\{R_{i}^{\prime}\right\}_{i \in I},\left\{p_{j}^{\prime}\right\}_{j \in J}\right)$ be fuzzy networks.

Then there is the greatest fuzzy relation $\varphi \in L^{A \times A^{\prime}}$ which satisfies (16), (17) and

$$
\varphi \circ p_{j}^{\prime} \leqslant p_{j}, \quad \varphi^{-1} \circ p_{j} \leqslant p_{j}^{\prime}, \quad \text { for all } j \in J .
$$

If, in addition, $\varphi$ satisfies

$$
p_{j} \leqslant \varphi \circ p_{j}^{\prime}, \quad p_{j}^{\prime} \leqslant \varphi^{-1} \circ p_{j}, \quad \text { for all } j \in J,
$$

then it is the greatest regular bisimulation between $\mathcal{N}$ and $\mathcal{N}^{\prime}$.

Otherwise, if $\varphi$ does not satisfy (19), then there is no any regular bisimulation between $\mathcal{N}$ and $\mathcal{N}^{\prime}$.

Proof. Evidently, the family of all fuzzy relations between $A$ and $A^{\prime}$ satisfying (16), (17) and (18) is non-empty, because it contains the empty relation between $A$ and $A^{\prime}$. By the same arguments used in the proof of Theorem 3.1 we obtain that the union $\varphi$ of all members of this family is the greatest fuzzy relation between $A$ and $A^{\prime}$ which satisfies (16), (17) and (18).

If $\varphi$ satisfies (19), then $\varphi$ is a regular bisimulation, and since any regular bisimulation between $\mathcal{N}$ and $\mathcal{N}^{\prime}$ must satisfy (16), (17) and (18), and $\varphi$ is the greatest fuzzy relation between $A$ and $A^{\prime}$ which satisfies these conditions, we conclude that $\varphi$ is the greatest regular bisimulation between $\mathcal{N}$ and $\mathcal{N}^{\prime}$.

Suppose that there exists a regular bisimulation $\psi$ between $\mathcal{N}$ and $\mathcal{N}^{\prime}$. Then $\psi$ satisfies (16), (17) and (18), whence $\psi \leqslant \varphi$, and consequently,

$$
p_{j}=\psi \circ p_{j}^{\prime} \leqslant \varphi \circ p_{j}^{\prime}
$$

and

$$
p_{j}^{\prime}=\psi^{-1} \circ p_{j} \leqslant \varphi^{-1} \circ p_{j}
$$

for every $j \in J$, which means that $\varphi$ satisfies (19). Therefore, if $\varphi$ does not satisfy (19), then there is no any regular bisimulation between $\mathcal{N}$ and $\mathcal{N}^{\prime}$.

Note that in the general case the fuzzy relation $\varphi$ from the previous theorem may be empty. However, if $\varphi$ satisfies (19), then it must be non-empty.

For fuzzy networks $\mathcal{N}=\left(A,\left\{R_{i}\right\}_{i \in I},\left\{p_{j}\right\}_{j \in J}\right)$ and $\mathcal{N}^{\prime}=\left(A^{\prime},\left\{R_{i}^{\prime}\right\}_{i \in I},\left\{p_{j}^{\prime}\right\}_{j \in J}\right)$, by $\mathcal{L}\left(\mathcal{N}, \mathcal{N}^{\prime}\right)$ we denote the subalgebra of $\mathcal{L}$ generated by all membership values taken by fuzzy relations and fuzzy sets from the families $\left\{R_{i}\right\}_{i \in I},\left\{R_{i}^{\prime}\right\}_{i \in I},\left\{p_{j}\right\}_{j \in J}$ and $\left\{p_{j}^{\prime}\right\}_{j \in J}$.

Now we prove a theorem which provides a procedure for testing the existence of a regular bisimulation between two fuzzy networks and computing the greatest one, in the case when it exists.
Theorem 3.3 Let $\mathcal{N}=\left(A,\left\{R_{i}\right\}_{i \in I},\left\{p_{j}\right\}_{j \in J}\right)$ and $\mathcal{N}^{\prime}=\left(A^{\prime},\left\{R_{i}^{\prime}\right\}_{i \in I},\left\{p_{j}^{\prime}\right\}_{j \in J}\right)$ be fuzzy networks, and let $\left\{\varphi_{k}\right\}_{k \in \mathbb{N}}$ be a sequence of fuzzy relations between $A$ and $A^{\prime}$ defined inductively as follows:

$$
\begin{aligned}
\varphi_{1} & =\bigwedge_{j \in J}\left(p_{j} \backslash p_{j}^{\prime}\right) \wedge\left(p_{j} / p_{j}^{\prime}\right), \\
\varphi_{k+1} & =\varphi_{k} \wedge \bigwedge_{i \in I}\left(\left[R_{i} \backslash\left(\varphi_{k} \circ R_{i}^{\prime}\right)\right] \wedge\left[\left(R_{i} \circ \varphi_{k}\right) / R_{i}^{\prime}\right]\right. \\
& \left.\wedge\left[\left(R_{i}^{\prime} \circ \varphi_{k}^{-1}\right) / R_{i}\right]^{-1} \wedge\left[R_{i}^{\prime} \backslash\left(\varphi_{k}^{-1} \circ R_{i}\right)\right]^{-1}\right),
\end{aligned}
$$

for each $k \in \mathbb{N}$. Then the following is true:

(a) the sequence $\left\{\varphi_{k}\right\}_{k \in \mathbb{N}}$ is decreasing;

(b) if there is $n \in \mathbb{N}$ such that $\varphi_{n+1}=\varphi_{n}$, then

(b1) $\varphi_{n}$ is the greatest regular fuzzy relation between $A$ and $A^{\prime}$ satisfying (16), (17) and $(18)$;

(b2) if $\varphi_{n}$ satisfies (19), then it is the greatest regular bisimulation between $\mathcal{N}$ and $\mathcal{N}^{\prime}$;

(b3) if $\varphi_{n}$ does not satisfy (19), there is no any regular bisimulation between $\mathcal{N}$ and $\mathcal{N}^{\prime}$;

(c) if $A$ and $A^{\prime}$ are finite sets and the subalgebra $\mathcal{L}\left(\mathcal{N}, \mathcal{N}^{\prime}\right)$ satisfies DCC, then $\left\{\varphi_{k}\right\}_{k \in \mathbb{N}}$ is a finite sequence and there exists $n \in \mathbb{N}$ such that $\varphi_{n+1}=\varphi_{n}$.

Proof. (a) It is clear that $\left\{\varphi_{k}\right\}_{k \in \mathbb{N}}$ is a decreasing sequence.

(b) First we note that for an arbitrary fuzzy relation $\varphi \in L^{A \times A^{\prime}}$ we have that $\varphi \leqslant \varphi_{1}$ if and only if

$$
\varphi \leqslant p_{j} \backslash p_{j}^{\prime} \text { and } \varphi \leqslant p_{j} / p_{j}^{\prime}
$$

for each $j \in J$, which is equivalent to

$$
\varphi^{-1} \circ p_{j}=p_{j} \circ \varphi \leqslant p_{j}^{\prime} \text { and } \varphi \circ p_{j}^{\prime} \leqslant p_{j},
$$

for each $j \in J$. This means that $\varphi$ satisfies the condition (18) if and only if $\varphi \leqslant \varphi_{1}$, and $\varphi_{1}$ is the greatest fuzzy relation between $A$ and $A^{\prime}$ which satisfies (18). Consequently, $\varphi_{k}$ satisfies (18) for every $k \in \mathbb{N}$.

Assume now that $\varphi_{n+1}=\varphi_{n}$ for some $n \in \mathbb{N}$. For each $i \in I$, by $\varphi_{n}=\varphi_{n+1} \leqslant R_{i} \backslash\left(\varphi_{n} \circ R_{i}^{\prime}\right)$ it follows $R_{i} \circ \varphi_{n} \leqslant \varphi_{n} \circ R_{i}^{\prime}$, and in a similar way we obtain that $\varphi_{n} \circ R_{i}^{\prime} \leqslant R_{i} \circ \varphi_{n}, \varphi_{n}^{-1} \circ R_{i} \leqslant R_{i}^{\prime} \circ \varphi_{n}^{-1}$ and $R_{i}^{\prime} \circ \varphi_{n}^{-1} \leqslant \varphi_{n}^{-1} \circ R_{i}$. Therefore, $\varphi_{n}$ satisfies (16), (17) and (18).

Let $\varphi \in L^{A \times A^{\prime}}$ be an arbitrary fuzzy relation satisfying (16), (17) and (18). Then $\varphi \leqslant \varphi_{1}$, since $\varphi_{1}$ is the greatest fuzzy relation satisfying (18). Suppose that $\varphi \leqslant \varphi_{k}$, for some $k \in \mathbb{N}$. Then for each $i \in I$ we have that

$$
R_{i} \circ \varphi=\varphi \circ R_{i}^{\prime} \leqslant \varphi_{k} \circ R_{i}^{\prime},
$$

whence it follows

$$
\varphi \leqslant R_{i} \backslash\left(\varphi_{k} \circ R_{i}^{\prime}\right) .
$$

In the same way we obtain that $\varphi \leqslant\left(R_{i} \circ \varphi_{k}\right) / R_{i}^{\prime}$, $\varphi \leqslant\left[\left(R_{i}^{\prime} \circ \varphi_{k}^{-1}\right) / R_{i}\right]^{-1}$ and $\varphi \leqslant\left[R_{i}^{\prime} \backslash\left(\varphi_{k}^{-1} \circ R_{i}\right)\right]^{-1}$, 
which means that $\varphi \leqslant \varphi_{k+1}$. Now, by induction we conclude that $\varphi \leqslant \varphi_{k}$, for every $k \in \mathbb{N}$, and consequently, $\varphi \leqslant \varphi_{n}$. Therefore, we have proved that $\varphi_{n}$ is the greatest fuzzy relation between $A$ and $A^{\prime}$ satisfying (16), (17) and (18).

The assertions (b2) and (b3) follow immediately by Theorem 3.2.

(c) Let $A$ and $A^{\prime}$ be finite sets and the subalgebra $\mathcal{L}\left(\mathcal{N}, \mathcal{N}^{\prime}\right)$ satisfy DCC. For all pairs $\left(a, a^{\prime}\right) \in A \times A^{\prime}$, we have that $\left\{\varphi_{k}\left(a, a^{\prime}\right)\right\}_{k \in \mathbb{N}}$ is a descending sequence in $\mathcal{L}\left(\mathcal{N}, \mathcal{N}^{\prime}\right)$. By the hypothesis, this sequence stabilizes, and since there are finitely many such sequences, we conclude that there is $n \in \mathbb{N}$ such that all these sequences stabilize after $n$ steps. Thus, the sequence $\left\{\varphi_{k}\right\}_{k \in \mathbb{N}}$ is finite and $\varphi_{n+1}=\varphi_{n}$ for $n \in \mathbb{N}$ whose existence has been established above.

This completes the proof of the theorem.

The previous theorem provides a procedure to test whether there is a regular bisimulation between two fuzzy networks, and in the case that it exists, the same procedure computes the greatest one. The procedure builds a descending sequence of fuzzy relations, in the way shown in Theorem 3.3 , and whenever a new member of the sequence is computed, the procedure checks whether it is equal to the previously computed members. The procedure terminates as soon as we find the first pair of equal consecutive members of the sequence, and in this case, the last computed member is the greatest fuzzy relation satisfying conditions (16), (17) and (18). Then, the procedure checks whether this fuzzy relation satisfies (19). If it satisfies that condition, then it is the greatest regular bisimulation between the given fuzzy networks. Otherwise, if it does not satisfy (19), then there is no any regular bisimulation between these fuzzy networks.

However, the proposed procedure do not necessarily terminate in a finite number of steps. The termination of the procedure depends on the underlying structure of membership values and on the membership values taken by the fuzzy relations and fuzzy sets that determine the considered fuzzy networks. Theorem 3.3 provides a sufficient condition for the termination of the procedure in a finite number of steps, when the subalgebra $\mathcal{L}\left(\mathcal{N}, \mathcal{N}^{\prime}\right)$ satisfies the descending chain condition. For instance, this condition is satisfied if $\mathcal{L}$ is a locally finite algebra, that is, if every finitely generated subalgebra of $\mathcal{L}$ is finite. The most known locally finite structures are the Boolean structure and the Gödel structure. For more information on local finiteness in t-norm based structures we refer to the recent paper [16].

Assume that $I, A$ and $B$ are finite sets and the subalgebra $\mathcal{L}\left(\mathcal{N}, \mathcal{N}^{\prime}\right)$ satisfies DCC. Let $c_{\otimes}, c_{\vee}, c_{\rightarrow}$ and $c_{\wedge}$ are computational costs of the operations $\otimes$, $\vee, \rightarrow$ and $\wedge$ in $\mathcal{L}$, and $|I|,|A|$ and $|B|$ the numbers of elements of $I, A$ and $B$. It can be shown that any single step in our procedure can be realized in time $O\left(|I| \cdot|A| \cdot|B| \cdot(|A|+|B|) \cdot\left(c_{\otimes}+c_{\vee}+c_{\rightarrow}+c_{\wedge}\right)\right)$. Values of the corresponding entries of $\left\{\varphi_{k}\right\}_{k \in \mathbb{N}}$ form
$|A| \cdot|B|$ descending chains of elements of $\mathcal{L}\left(\mathcal{N}, \mathcal{N}^{\prime}\right)$, so there is $l \in \mathbb{N}$ such that the number of different elements in each of these chains is less than or equal to $l$. Thus, the number of steps in our procedure is $O(l \cdot|A| \cdot|B|)$ and the total computation time for the whole procedure is

$O\left(l \cdot|I| \cdot|A|^{2} \cdot|B|^{2} \cdot(|A|+|B|) \cdot\left(c_{\otimes}+c_{\vee}+c_{\rightarrow}+c_{\wedge}\right)\right)$.

Note that the number $l$ is a characteristic of the sequence $\left\{\varphi_{k}\right\}_{k \in \mathbb{N}}$ and in general it is not a characteristic of the algebra $\mathcal{L}\left(\mathcal{N}, \mathcal{N}^{\prime}\right)$. However, sometimes the number of different elements in all descending chains in $\mathcal{L}\left(\mathcal{N}, \mathcal{N}^{\prime}\right)$ may have an upper bound $l$. For example, if $\mathcal{L}\left(\mathcal{N}, \mathcal{N}^{\prime}\right)$ is finite, then we can assume that $l$ is its number of elements. In particular, if $\mathcal{L}$ is the Gödel structure, then the only values that can be taken by fuzzy relations $\left\{\varphi_{k}\right\}_{k \in \mathbb{N}}$ are 1 and those taken by fuzzy sets and relations that determine $\mathcal{N}$ and $\mathcal{N}^{\prime}$, so the number of these values is finite. If this number is denoted by $j$, then the total computation time of the procedure is $O\left(j \cdot|I| \cdot|A|^{2} \cdot|B|^{2} \cdot(|A|+|B|)\right)$ (here the operations $\otimes, \vee, \rightarrow$ and $\wedge$ can be performed in constant time). If $\mathcal{L}$ is the Boolean structure, then $j=2$ and the procedure works in time $O\left(|I| \cdot|A|^{2} \cdot|B|^{2} \cdot(|A|+|B|)\right)$.

According to Theorem 3.1, if there exists at least one regular bisimulation between two fuzzy networks, then there exists the greatest one, and it is a partial fuzzy function. However, if we want to establish some kind of structural similarity between two fuzzy networks, it is necessary that every actor of the first network is similar to some actor of the second one, and vice versa. In other words, those regular bisimulations which are surjective $\mathcal{L}$-functions are especially interesting. It is easy to check that if there is a regular bisimulation between two fuzzy networks which is a surjective $\mathcal{L}$-function, then the greatest one also has this property, i.e., it is a uniform fuzzy function. Consequently, we find that it is very interesting to study those regular bisimulations which are uniform fuzzy relations.

The next theorem shows that a uniform regular bisimulation between two fuzzy networks determines a pair of regular equivalences on these networks and an isomorphism between the corresponding quotient fuzzy networks (i.e., an isomorphism between the positions or roles in these networks), and vice versa.

Theorem 3.4 Let $\mathcal{N}=\left(A,\left\{R_{i}\right\}_{i \in I},\left\{p_{j}\right\}_{j \in J}\right)$ and $\mathcal{N}^{\prime}=\left(A^{\prime},\left\{R_{i}^{\prime}\right\}_{i \in I},\left\{p_{j}^{\prime}\right\}_{j \in J}\right)$ be fuzzy networks and let $\varphi \in L^{A \times A^{\prime}}$ be a uniform fuzzy relation. Then $\varphi$ is a regular bisimulation between $\mathcal{N}$ and $\mathcal{N}^{\prime}$ if and only if the following conditions hold:

(i) the kernel of $\varphi$ is a regular equivalence on $\mathcal{N}$;

(ii) the cokernel of $\varphi$ is a regular equivalence on $\mathcal{N}^{\prime}$;

(iii) $\bar{\varphi}$ is an isomorphism of quotient networks $\mathcal{N} / E_{A}^{\varphi}$ and $\mathcal{N}^{\prime} / E_{A^{\prime}}^{\varphi}$ 
Proof. Let $E_{A}^{\varphi}=\alpha$ and $E_{A^{\prime}}^{\varphi}=\alpha^{\prime}$. By Theorem 4.2 [7] it follows that $\alpha=\varphi \circ \varphi^{-1}$ and $\alpha^{\prime}=\varphi^{-1} \circ \varphi$, and for all $\psi \in C R(\varphi), a, b \in A$ and $a^{\prime} \in A^{\prime}$ we have

$$
\left.\varphi(a, \psi(b))=\alpha(a, b) \text { and } \varphi\left(a, a^{\prime}\right)=\alpha^{\prime}\left(\psi(a), a^{\prime}\right)\right)
$$

Suppose that $\varphi$ is a regular bisimulation. Then for each $i \in I$ we have that

$$
\begin{aligned}
\alpha \circ R_{i} & =\varphi \circ \varphi^{-1} \circ R_{i}=\varphi \circ R_{i}^{\prime} \circ \varphi^{-1} \\
& =R_{i} \circ \varphi \circ \varphi^{-1}=R_{i} \circ \alpha,
\end{aligned}
$$

and for each $j \in J$ we have that

$$
p_{j} \circ \alpha=p_{j} \circ \varphi \circ \varphi^{-1} \leqslant p_{j}^{\prime} \circ \varphi^{-1} \leqslant p_{j} \leqslant p_{j} \circ \alpha,
$$

whence $p_{j} \circ \alpha=p_{j}$. Thus, $\alpha$ is a regular equivalence on $\mathcal{N}$. In the same way we show that $\alpha^{\prime}$ is a regular bisimulation on $\mathcal{N}^{\prime}$.

Next, for arbitrary $i \in I, a, b \in A$ and $\psi \in C R(\varphi)$ we have that

$$
\begin{aligned}
\bar{R}_{i}^{\prime}\left(\bar{\varphi}\left(\alpha_{a}\right), \bar{\varphi}\left(\alpha_{b}\right)\right)=\bar{R}_{i}^{\prime}\left(\alpha_{\psi(a)}^{\prime}, \alpha_{\psi(b)}^{\prime}\right) \\
=\left(\alpha^{\prime} \circ R_{i}^{\prime} \circ \alpha^{\prime}\right)(\psi(a), \psi(b)) \\
=\left(\varphi^{-1} \circ R_{i} \circ \varphi\right)(\psi(a), \psi(b)) \\
=\bigvee_{c, d \in A} \varphi^{-1}(\psi(a), c) \otimes R_{i}(c, d) \otimes \varphi(d, \psi(b)) \\
=\bigvee_{c, d \in A} \alpha(a, c) \otimes R_{i}(c, d) \otimes \alpha(d, b) \\
=\left(\alpha \circ R_{i} \circ \alpha\right)(a, b) \\
=\bar{R}_{i}\left(\alpha_{a}, \alpha_{b}\right) .
\end{aligned}
$$

Also, for each $j \in J$ by (10) and (11) it follows

$$
\varphi^{-1} \circ p_{j} \leqslant \varphi^{-1} \circ \varphi \circ p_{j}^{\prime}=\alpha^{\prime} \circ p_{j}^{\prime}=p_{j}^{\prime} \leqslant \varphi^{-1} \circ p_{j},
$$

so $\varphi^{-1} \circ p_{j}=\alpha^{\prime} \circ p_{j}^{\prime}$, and for all $j \in J, a \in A$ and $\psi \in C R(\varphi)$ we obtain that

$$
\begin{aligned}
\bar{p}_{j}^{\prime}\left(\bar{\varphi}\left(\alpha_{a}\right)\right) & =\bar{p}_{j}^{\prime}\left(\alpha_{\psi(a)}^{\prime}\right)=\left(\alpha^{\prime} \circ p_{j}^{\prime}\right)(\psi(a)) \\
& =\left(\varphi^{-1} \circ p_{j}\right)(\psi(a)) \\
& \left.=\bigvee_{b \in A} \varphi^{-1}(\psi(a), b)\right) \otimes p_{j}(b) \\
& \left.=\bigvee_{b \in A} \alpha(a, b)\right) \otimes p_{j}(b) \\
& =\left(\alpha \circ p_{j}\right)(a)=\bar{p}_{j}\left(\alpha_{a}\right) .
\end{aligned}
$$

Therefore, $\bar{\varphi}$ is an isomorphism of quotient networks $\mathcal{N} / E_{A}^{\varphi}$ and $\mathcal{N}^{\prime} / E_{A^{\prime}}^{\varphi}$

Conversely, assume that (i), (ii) and (iii) hold.

For all $j \in J, a \in A$ and $\psi \in C R(\varphi)$ we have that

$$
\begin{aligned}
p_{j}(a) & =\left(p_{j} \circ \alpha\right)(a)=\bar{p}_{j}\left(\alpha_{a}\right)=\bar{p}_{j}^{\prime}\left(\bar{\varphi}\left(\alpha_{a}\right)\right) \\
& =\bar{p}_{j}^{\prime}\left(\alpha_{\psi(a)}^{\prime}\right)=\left(p_{j}^{\prime} \circ \alpha^{\prime}\right)(\psi(a)) \\
& =\bigvee_{a^{\prime} \in A^{\prime}} p_{j}^{\prime}\left(a^{\prime}\right) \otimes \alpha^{\prime}\left(a^{\prime}, \psi(a)\right) \\
& =\bigvee_{a^{\prime} \in A^{\prime}} p_{j}^{\prime}\left(a^{\prime}\right) \otimes \varphi\left(a, a^{\prime}\right)=\left(\varphi \circ p_{j}^{\prime}\right)(a),
\end{aligned}
$$

so $p_{j}=\varphi \circ p_{j}^{\prime}=p_{j}^{\prime} \circ \varphi^{-1}$. In the same way, using $\varphi^{-1}$ instead of $\varphi$, we obtain that $p_{j}^{\prime}=\varphi^{-1} \circ p_{j}=p_{j} \circ \varphi$.

On the other hand, using the fact that $\alpha$ and $\alpha^{\prime}$ are regular fuzzy equivalences on $\mathcal{N}$ and $\mathcal{N}^{\prime}$, respectively, $\varphi=\varphi \circ \varphi^{-1} \circ \varphi=\alpha \circ \varphi=\varphi \circ \alpha^{\prime}, \alpha \circ \alpha=\alpha$ and $\alpha^{\prime} \circ \alpha^{\prime}=\alpha^{\prime}$, for an arbitrary $i \in I$ we obtain

$$
R_{i} \circ \varphi=R_{i} \circ \alpha \circ \alpha \circ \varphi=\alpha \circ R_{i} \circ \alpha \circ \varphi,
$$

and similarly,

$$
\alpha^{\prime} \circ R_{i}^{\prime} \circ \alpha^{\prime}=\alpha^{\prime} \circ R_{i}^{\prime} .
$$

Moreover, for all $a, b \in A$ and $\psi \in C R(\varphi)$ we obtain

$$
\begin{aligned}
& \left(\alpha \circ R_{i} \circ \alpha\right)(a, b)=\bar{R}_{i}\left(\alpha_{a}, \alpha_{b}\right) \\
& \quad=\bar{R}_{i}^{\prime}\left(\bar{\varphi}\left(\alpha_{a}\right), \bar{\varphi}\left(\alpha_{b}\right)\right)=\bar{R}_{i}^{\prime}\left(\alpha_{\psi(a)}^{\prime}, \alpha_{\psi(b)}^{\prime}\right) \\
& \quad=\left(\alpha^{\prime} \circ R_{i}^{\prime} \circ \alpha^{\prime}\right)(\psi(a), \psi(b)) .
\end{aligned}
$$

Now, for arbitrary $a \in A, a^{\prime} \in A^{\prime}$ and $\psi \in C R(\varphi)$ we have that

$$
\begin{aligned}
\left(R_{i} \circ \varphi\right) & \left(a, a^{\prime}\right)=\left(\alpha \circ R_{i} \circ \alpha \circ \varphi\right)\left(a, a^{\prime}\right) \\
= & \bigvee_{b \in A}\left(\alpha \circ R_{i} \circ \alpha\right)(a, b) \otimes \varphi\left(b, a^{\prime}\right) \\
= & \bigvee_{b \in A}\left(\alpha^{\prime} \circ R_{i}^{\prime} \circ \alpha^{\prime}\right)(\psi(a), \psi(b)) \otimes \alpha^{\prime}\left(\psi(b), a^{\prime}\right) \\
\leqslant & \bigvee_{b^{\prime} \in A^{\prime}}\left(\alpha^{\prime} \circ R_{i}^{\prime} \circ \alpha^{\prime}\right)\left(\psi(a), b^{\prime}\right) \otimes \alpha^{\prime}\left(b^{\prime}, a^{\prime}\right) \\
= & \left(\alpha^{\prime} \circ R_{i}^{\prime} \circ \alpha^{\prime} \circ \alpha^{\prime}\right)\left(\psi(a), a^{\prime}\right) \\
= & \left(\alpha^{\prime} \circ R_{i}^{\prime}\right)\left(\psi(a), a^{\prime}\right) \\
= & \bigvee_{c^{\prime} \in A^{\prime}} \alpha^{\prime}\left(\psi(a), c^{\prime}\right) \otimes R_{i}^{\prime}\left(c^{\prime}, a^{\prime}\right) \\
= & \bigvee_{c^{\prime} \in A^{\prime}} \varphi\left(a, c^{\prime}\right) \otimes R_{i}^{\prime}\left(c^{\prime}, a^{\prime}\right) \\
= & \left(\varphi \circ R_{i}^{\prime}\right)\left(a, a^{\prime}\right),
\end{aligned}
$$

and thus, $R_{i} \circ \varphi \leqslant \varphi \circ R_{i}^{\prime}$. In a similar way, using $\varphi^{-1}$ and $(\bar{\varphi})^{-1}$ instead of $\varphi$ and $\bar{\varphi}$, we prove the opposite inequality. Hence, $R_{i} \circ \varphi=\varphi \circ R_{i}^{\prime}$. Also, in a similar way we prove that $\varphi^{-1} \circ R_{i}=R_{i}^{\prime} \circ \varphi^{-1}$.

Therefore, we have proved that $\varphi$ is a regular bisimulation between networks $\mathcal{N}$ and $\mathcal{N}^{\prime}$.

\section{Concluding remarks}

In this paper we have defined two types of simulations and five types of bisimulations for fuzzy social networks, but due to limitations in the length of the paper, we have discussed only one of them - regular bisimulations. In our further research we intend to perform a deeper study of all types of simulations and bisimulations. This is particularly important if we have in mind an example provided by Brynielsson et al. [4], which shows that the so-called simulation equivalences can identify social positions that cannot be identified by means of regular equivalences. Consequently, which type of equivalence relations or bisimulations is interesting to consider depends on the problem at hand, and it is necessary 
to consider several different types of these relations for given networks, in order to understand them it completely.

\section{Acknowledgement}

This research was supported by the Ministry of Education, Science and Technological Development, Republic of Serbia, Grant No. 174013.

\section{References}

[1] V. Batagelj, Social Network Analysis, LargeScale, in R. A. Meyers (Ed.), Encyclopedia of Complexity and Systems Science, Springer, New York, pp. 8245-8265, 2009.

[2] R. Bělohlávek, Fuzzy Relational Systems: Foundations and Principles, Kluwer, New York, 2002.

[3] R. Bělohlávek and V. Vychodil, Fuzzy Equational Logic, Studies in Fuzziness and Soft Computing, Springer, Berlin-Heidelberg, 2005.

[4] J. Brynielsson, L. Kaati and P. Svenson, Social positions and simulation relations, Social Network Analysis and Mining, 2:39-52, Springer, 2012.

[5] M. Ćirić and S. Bogdanović, Fuzzy social network analysis, Godišnjak Učiteljskog fakulteta u Vranju, 1:179-190, University of Niš, Teachers Training Faculty Vranje, 2010.

[6] M. Ćirić, J. Ignjatović and S. Bogdanović, Uniform fuzzy relations and fuzzy functions, Fuzzy Sets and Systems, 160:1054-1081, Elsevier, 2009.

[7] M. Ćirić, J. Ignjatović, N. Damljanović and M. Bašić, Bisimulations for fuzzy automata, Fuzzy Sets and Systems, 186:100-139, Elsevier, 2012.

[8] M. Ćirić, J. Ignjatović, I. Jančić and N. Damljanović, Computation of the greatest simulations and bisimulations between fuzzy automata, Fuzzy Sets and Systems, 208:22-42, Elsevier, 2012.

[9] M. Ćirić, J. Ignjatović, M. Bašić and I. Jančić, Nondeterministic automata: Equivalence, bisimulations, and uniform relations, Information Sciences, 216:185-218, Elsevier, 2014.

[10] M. Ćirić, A. Stamenković, J. Ignjatović and T. Petković, Fuzzy relation equations and reduction of fuzzy automata, Journal of Computer and System Sciences, 76:609-633, Elsevier, 2010.

[11] P. Doreian, Positional analysis and blockmodeling, in: R. A. Meyers (Ed.), Encyclopedia of Complexity and Systems Science, Springer, New York, pp. 6913-6927, 2009.

[12] P. Doreian, V. Batagelj and A. Ferligoj, Generalized Blockmodeling, Cambridge University Press, Cambridge, 2005.
[13] T.F. Fan and C.J. Liau, Logical characterizations of regular equivalence in weighted social networks, Artiffcial Intelligence 214:66-88, Elsevier, 2014.

[14] T.F. Fan, C.J. Liau and T.Y. Lin, Positional analysis in fuzzy social networks, in: Proceedings of the 3rd IEEE International Conference on Granular Computing, pp 423-428, IEEE, 2007.

[15] T.F. Fan, C.J. Liau and T.Y. Lin, A theoretical investigation of regular equivalences for fuzzy graphs, International Journal of Approximate Reasoning 49(3):678-688, Elsevier, 2008.

[16] S. Gottwald, Local and relativized local finiteness in t-norm based structures, Information Sciences 228:26-36, Elsevier, 2013.

[17] J. Ignjatović and M. Ćirić, Weakly linear systems of fuzzy relation inequalities and their applications: A brief survey, Filomat, 26(2):207241, University of Niš, Faculty of Science and Mathematics, 2012.

[18] J. Ignjatović, M. Ćirić and S. Bogdanović, On the greatest solutions to weakly linear systems of fuzzy relation inequalities and equations, Fuzzy Sets and Systems 161:3081-3113, Elsevier, 2010.

[19] J. Ignjatović, M. Ćirić, N. Damljanović and I. Jančić, Weakly linear systems of fuzzy relation inequalities: The heterogeneous case, Fuzzy Sets and Systems, 199:64-91, Elsevier, 2012.

[20] F. Lorrain and H. C. White, Structural equivalence of individuals in social networks, Journal of Mathematical Sociology, 1:49-80, Taylor \& Francis, 1971.

[21] M. Marx and M. Masuch, Regular equivalence and dynamic logic, Social Networks, 25(1):5165, Elsevier, 2003.

[22] R. Milner, A calculus of communicating systems, Lecture Notes in Computer Science, vol. 92, Springer, 1980.

[23] D. Park, Concurrency and automata on infinite sequences, in: P. Deussen (ed.), Theoretical Computer Science - 5th GI-Conference Karlsruhe, March 23-25, 1981, pp. 167-183, Lecture Notes in Computer Science 104, Springer, 1981.

[24] J. Scott, Social Network Analysis, Overview of, in: R. A. Meyers (Ed.), Encyclopedia of Complexity and Systems Science, Springer, New York, pp. 8265-8279, 2009.

[25] A. Stamenković, M. Ćirić and J. Ignjatović, Reduction of fuzzy automata by means of fuzzy quasi-orders, Information Sciences, 275:168198, Elsevier, 2014.

[26] D. R. White and K. P. Reitz, Graph and semigroup homomorphisms on networks and relations, Social Networks, 5:143-234, Elsevier, 1983. 